\title{
Clostridium difficile: I 8 months surveillance at the Niguarda Hospital - Milano
}

\author{
Diana Fanti, Antonella Barzaghi, Maria Chiara Sironi, Monica Drago, Chiara Vismara, \\ Giuseppe Ortisi \\ S.C. Microbiologia e Virologia, Dipartimento di Medicina di Laboratorio, A.O. Ospedale Niguarda Ca' Granda
}

Key words: Clostridium difficile, MLST, surveillance

Clostridium difficile: 18 mesi di sorveglianza presso A.O. Niguarda - Milano

\section{SUMMARY}

Clostridium difficile is currently one of the most common cause of diarrhea in hospitalized or residents in long term care institutions patients, symptoms of infection ranging from diarrhea to pseudo-membranous colitis and toxic megacolon. The disease is due to the production of enterotoxin A, cytotoxin B or binary toxin.

The emergence of hypervirulent new strains showing a tcdC regulatory gene deletion (ribotype 027) has been observed in recent years.

Stool or rectal swab specimens were screened using automated real-time multiplex PCR (GeneXpert, Cellbio) to detect the presence of toxins producing ( $B$ toxin, binary toxin and tcdC gene deletion) $C$. difficile.

All positive samples were cultured in order to isolate the causing infection strains. The binary toxin and/or tcdC deletion producing strains were genotyped using Multilocus Sequence Typing (MLST).

MLST compares the intragenic sequences of seven housekeeping genes and provides a unique combination of alleles; to each combination is then assigned a Sequence Typing (ST).

In the period January 2010 - June 20I I, 368I samples from 2234 patients were analyzed.

Four hundred seventy-three patients (2I.2\%) were positive for the presence of $C$. difficile, 34 (7.2\%) of there were also positive for binary toxin and 3 were positive for the tcdC gene deletion (suspected C. difficile NAP027). All the strains having tcdC gene deletion, showes ST3 typing by MLST method. These strains were isolated during the same period. The three patiens were epidemiologically linked: the patient $A$ was hospitalized in the same room of patient $B$, while patient $C$ was managed by the same team care of patient $A$. The spread ofsuch $C$. difficile strains is at the present very low in Italy.

Our data confirm the poor prevalence of ribotype NAP027 and the usefulness of a presumptive diagnosis to implement immediately appropriate control measures.

\section{INTRODUZIONE}

Clostridium difficile rappresenta la principale causa di diarrea in pazienti ospedalizzati o residenti in istituti di lungodegenza.

I quadri clinici possono comprendere diarrea, colite pseudomembranosa e, talvolta, megacolon tossico. La malattia è dovuta alla produzione di Enterotossina (tossina A - TcdA) e di Citotossina (tossina B - TcdB).

Negli ultimi anni è stata osservata l'emergenza di ceppi iper-virulenti che presentano una delezione nel gene regolatore tcdC (NAP1/O27) (1).

\section{MATERIALI E METODI}

I campioni di feci o i tamponi rettali sono stati esaminati utilizzando una real-time multiplex PCR automatizzata (GeneXpert, Cellbio) per la rilevazione della presenza di $C$. difficile tossinogenico (tossina B, binaria e la delezione nel gene tcdC). Tutti i campioni positivi sono stati coltivati per isolare il ceppo responsabile dell'infezione.

I ceppi produttori di tossina binaria e/o con delezione di tcdC sono stati sottoposti a genotipizzazione mediante Multilocus Sequence Typing (MLST), che prevede il confronto fra le sequenze intrageniche di sette geni housekeeping. In base a tale metodo si giunge ad una combinazione unica di alleli a cui è assegnato un determinato Sequence Type (ST).

\section{Geni housekeeping:}

- $\operatorname{aroE}$ (shimate dehydrogenase)

- dutA (dUTP pyrophosphatase)

- gmk (gualynate kinase)

- groEL (60 kDa chaperonin)

- $\operatorname{rec} A$ (recombinase)

- $\operatorname{sodA}$ (superoxide dismutase)

- tpi (triosephosphate isomerase)

(Le sequenze dei primer utilizzati per il sequen-

\section{Corresponding author: Diana Fanti}

S.C. Microbiologia e Virologia, Dipartimento di Medicina di Laboratorio, A.O. Ospedale Niguarda Ca' Granda Piazza Ospedale Maggiore, 3 - 20162 Milano - Tel.: 0264443886 - Fax: 0264443680

E-mail: diana.fanti@ospedaleniguarda.it 
ziamento sono disponibili sul sito:

http://www.pasteur.fr/recherche/genopole/PF8/ml st/Cdifficile2.html)

\section{RISULTATI}

Nel periodo da gennaio 2010 a giugno 2011 sono stati analizzati 3681 campioni da 2234 pazienti. 473 pazienti $(21.2 \%)$ sono risultati positivi per la presenza di $C$. difficile; di questi 34 (7.2\%) erano positivi anche per tossina binaria e 3 delezione nel gene tcdC (sospetto $C$. difficile NAP1/O27) (Figura I).

I ceppi con delezione nel gene tcdC, tipizzati tramite metodica MLST, sono risultati tutti ST3, mostrando il profilo: aroE (3); dutA (3); gmk (3); groEL (9); recA (1); $\operatorname{sodA}(1)$; tpi (3). Tali ceppi, isolati nello stesso periodo di tempo, sono inoltre risultati resistenti ai chinoloni (CMI ciprofloxacina: $128 \mathrm{mg} / \mathrm{L}$ ).

I tre pazienti erano correlati dal punto di vista epidemiologico: il paziente A era ricoverato nella stessa stanza del paziente $B$; mentre il paziente $C$ era seguito dallo stesso team di cura del paziente. A.

\section{CONCLUSIONI}

La diffusione dei ceppi di $C$. difficile in Italia è per ora molto contenuta. Il profilo ST3 dei ceppi analizzati è identico al ST del ceppo ipervirulento NAP1/O27 (2-3). I nostri dati confermano la scarsa diffusione del ribotipo NAP027 e l'utilità di una diagnosi presuntiva immediata per implementare adeguate misure di contenimento.

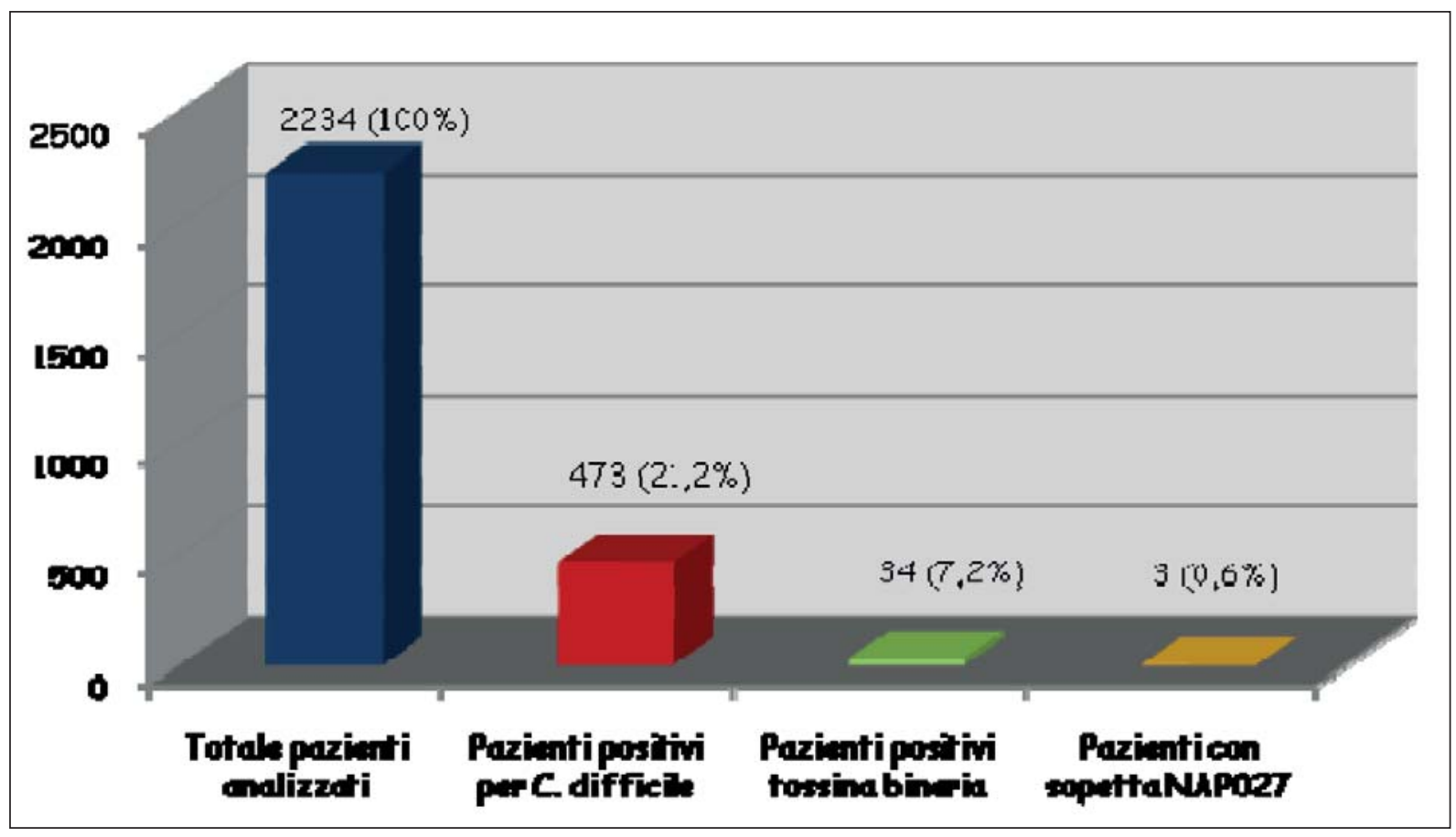

Figura I. Periodo di sorveglianza Clostridium difficile: gennaio 2010 - giugno 201 I.

\section{BIBLIOGRAFIA}

1. Baldan R, Cavallerio P, Tuscano A, et al. First report of Hypervirulent strains polymerase chain reaction ribotypes 027 and 078 causing severe Clostridium difficile infection in Italy. Clinical Infectious Diseases 2010; 50: 126-7.
2. Clements ACA, Soares Magalhães RJ, Tatem AJ, et al. Clostridium difficile PCR ribotype 027: assessing the risks of further worldwide spread. Lancet Infect Dis 2010; 10: 395-404

3. Lemée L, Pons JL. Multilocus Sequence Typing for Clostridium difficile. Methods Mol Biol. 2010; 646: 77-90. 